\title{
Advanced Chemical Analysis Using an Annular Four-Channel Silicon Drift Detector
}

\author{
Ralf Terborg, ${ }^{1 *}$ Andi Kaeppel, ${ }^{1}$ Baojun Yu, ${ }^{2}$ Max Patzschke, ${ }^{1}$ Tobias Salge, ${ }^{3}$ and Meiken Falke ${ }^{1}$ \\ ${ }^{1}$ Bruker Nano GmbH, Am Studio 2D, 10317 Berlin, Germany \\ ${ }^{2}$ Bruker Nano Analytics Division, 19F, Xinyuan Technology Building, No.418 Guiping Rd, Caohejing Development Zone, \\ Shanghai 200233, China \\ ${ }^{3}$ Natural History Museum, Cromwell Road, London SW7 5BD, UK \\ ^ralf.terborg@bruker.com
}

\begin{abstract}
Successful energy-dispersive analysis with silicon drift detectors (SDDs) spurred further instrumentation development, leading to multi-channel SDDs with four separate detector segments integrated into a single SDD chip. The use of multiple channels provides greater geometrical X-ray collection efficiency and allows higher throughput. We demonstrate the use of an annular multi-channel detector in the scanning electron microscope. The detector is placed between the pole piece and the sample. Optimization of acquisition parameters for various problems of materials and life science is explained. The advantages for analyses at low beam current, high speed, and for highly topographic samples are shown.
\end{abstract}

\section{Introduction}

Peltier-cooled silicon drift detectors (SDDs) have become standard instrumentation for energy-dispersive X-ray spectrometry (EDS) in electron microscopes [1,2]. State-of-the-art SDDs reach an energy resolution of $121 \mathrm{eV}$ at $\mathrm{Mn}-\mathrm{K} \alpha$, show good low-energy performance below $100 \mathrm{eV}$, and produce Gaussian peak shapes undistorted by detector artifacts such as shelf and tail effects. This is beneficial for peak separation over the entire available energy range. But peak separation is particularly important for overlaps of low-energy lines, such as the K-lines of light elements (Be - F) and the L-lines $(\mathrm{K}-\mathrm{Ni})$, M-lines $(\mathrm{Mo}-\mathrm{La})$, and N-lines ( Hf - U) of heavier elements.

The use of multiple detectors allows a substantially higher acquisition speed and higher throughput because of greater geometrical collection efficiency from a larger solid angle for $\mathrm{X}$-ray collection. Such multiple detector arrangements need multiple accessible EDS ports, which may not be possible because of geometric limitations inside and outside the microscope. Thus, further increasing the solid angle and throughput by using more than two individual detectors becomes impractical. Fortunately, thanks to advances in SDD technology, other alternatives are available.

Multi-channel SDDs with four separate detector segments integrated into one single SDD chip have been developed to deliver improved EDS performance. The combined parallel readout of the detector segments provides the increased count rate that would be expected in the case of several individual detectors. The detection geometry can be optimized using an annular arrangement of the SDD segments, as implemented in the annular Bruker XFlash ${ }^{\circledR}$ FlatQUAD system.

The present article shows this detector optimized for different tasks. We show results of this dealing with the challenges of meteorite exploration, polymer research, and low-yield life science samples.

\section{Materials and Methods}

The annular detector and its setup. The four integrated SDD segments of the XFlash ${ }^{\circledR}$ FlatQUAD detector are arranged in radial symmetry around a hole through which the electron beam passes. The detector is inserted horizontally and placed like a backscattered electron (BSE) detector between sample and pole piece. Thus the annular active SDD area resides directly above and very close to the sample, achieving both a high take-off angle between $40^{\circ}$ and $70^{\circ}$, which minimizes shadowing effects, and a relatively large solid angle of collection. Each of the four SDD segments has an active area of $15 \mathrm{~mm}^{2}$, resulting in a total of $60 \mathrm{~mm}^{2}$. The respective annular geometry (Figure 1) delivers a solid angle of more than 1 steradian (sr) [3-6]. To avoid alteration of X-ray spectroscopy results by electrons coming from the sample and to maintain low-energy X-ray sensitivity, three polymer windows of different thickness can be placed semiautomatically in front of the chip depending on the acceleration voltage used. Because this annular SDD array is closer to the specimen, the solid angle value is 100 times higher than that typically available in a $10 \mathrm{~mm}^{2}$ detector with a $35^{\circ}$ takeoff angle and over 10 times higher than that available from two $30 \mathrm{~mm}^{2}$ detectors or a single $100 \mathrm{~mm}^{2}$ detector in conventional setups. High count rates can be obtained even with low probe currents. This is achieved by processing the counts from the annular detector through four separate electronic channels in parallel, leading to a maximum output count rate of up to 2,400 kilo-counts per second (kcps) $[3,7]$.

These advantages invite challenging analysis approaches such as the use of low electron accelerating voltages, ultra-low

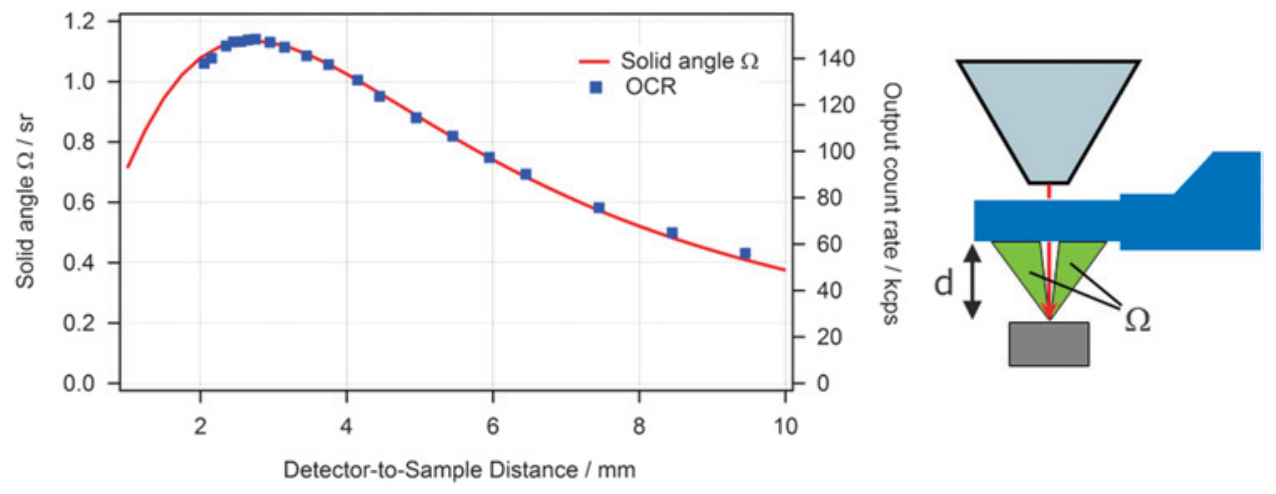

Figure 1: Left: Solid angle $\Omega$ and X-ray output count rate (OCR) versus detector-to-sample distance. Note that the optimum detector-sample distance $d$ of $2.8 \mathrm{~mm}$ delivers a solid angle of $>1.1 \mathrm{sr}$. Right: Detector (blue) arrangement between SEM pole piece and sample. 


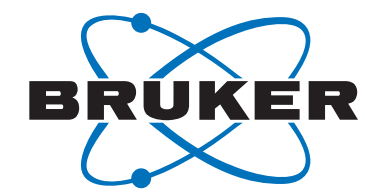

\section{Someone has to be first. XFlash ${ }^{\circledR}$ FlatQUAD, multiple detector systems \& VZ}

\section{Analyze textured samples with ease.}
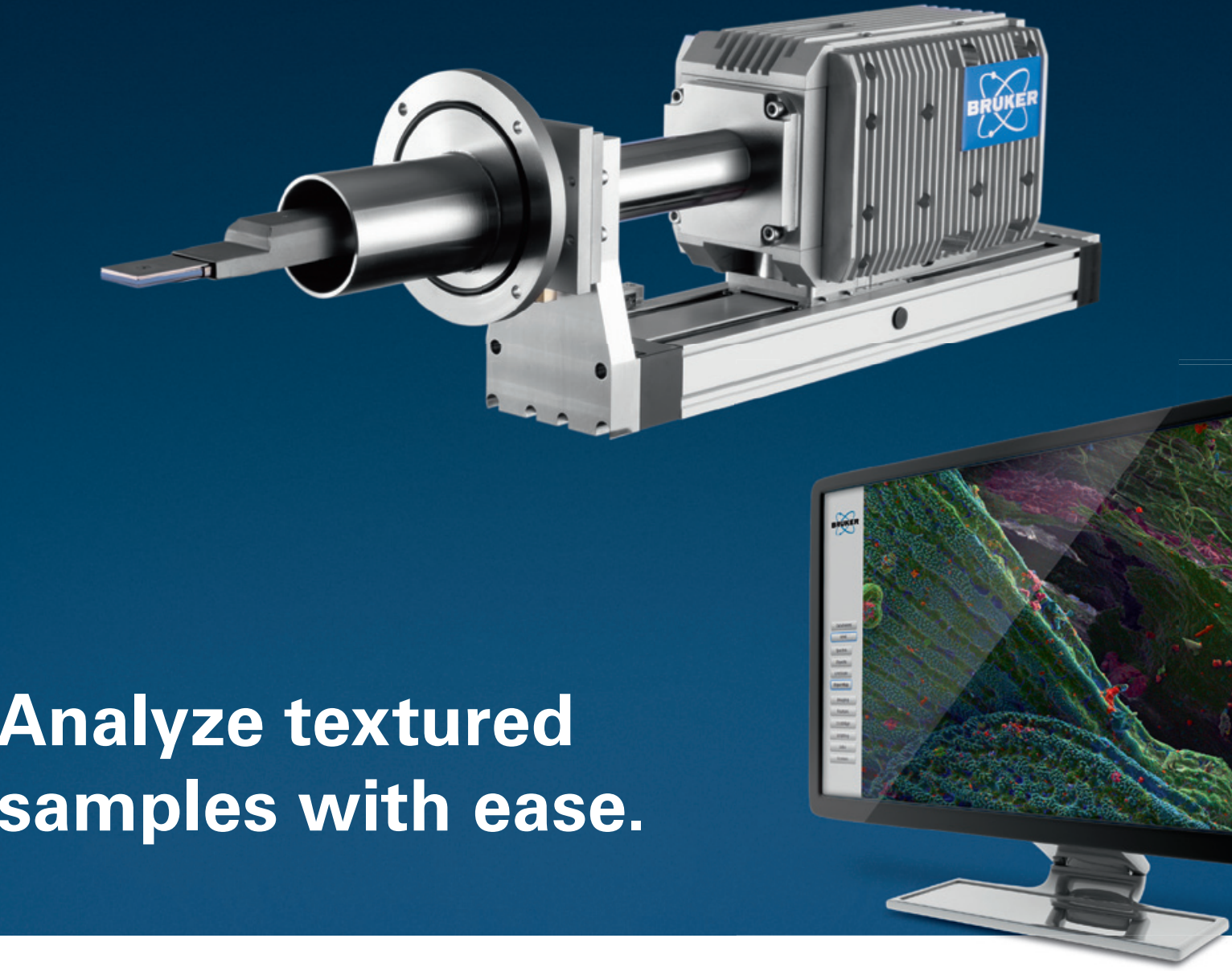

- The unique XFlash ${ }^{\circledR}$ FlatQUAD offers a large solid angle and an amazing take-off angle.

This minimizes shadowing so you can see every nook and cranny of your sample in record time.

- Bruker's multiple detector systems provide large solid angles and great take-off angles too. You can start with one detector building up to four, depending on your needs.

- The Variable Z (VZ) adapter allows you to optimize take-off angles in-situ, which significantly improves the analysis of topographically challenging samples.

Someone has to be first.

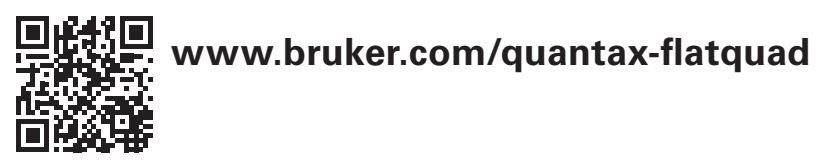

Innovation with Integrity 

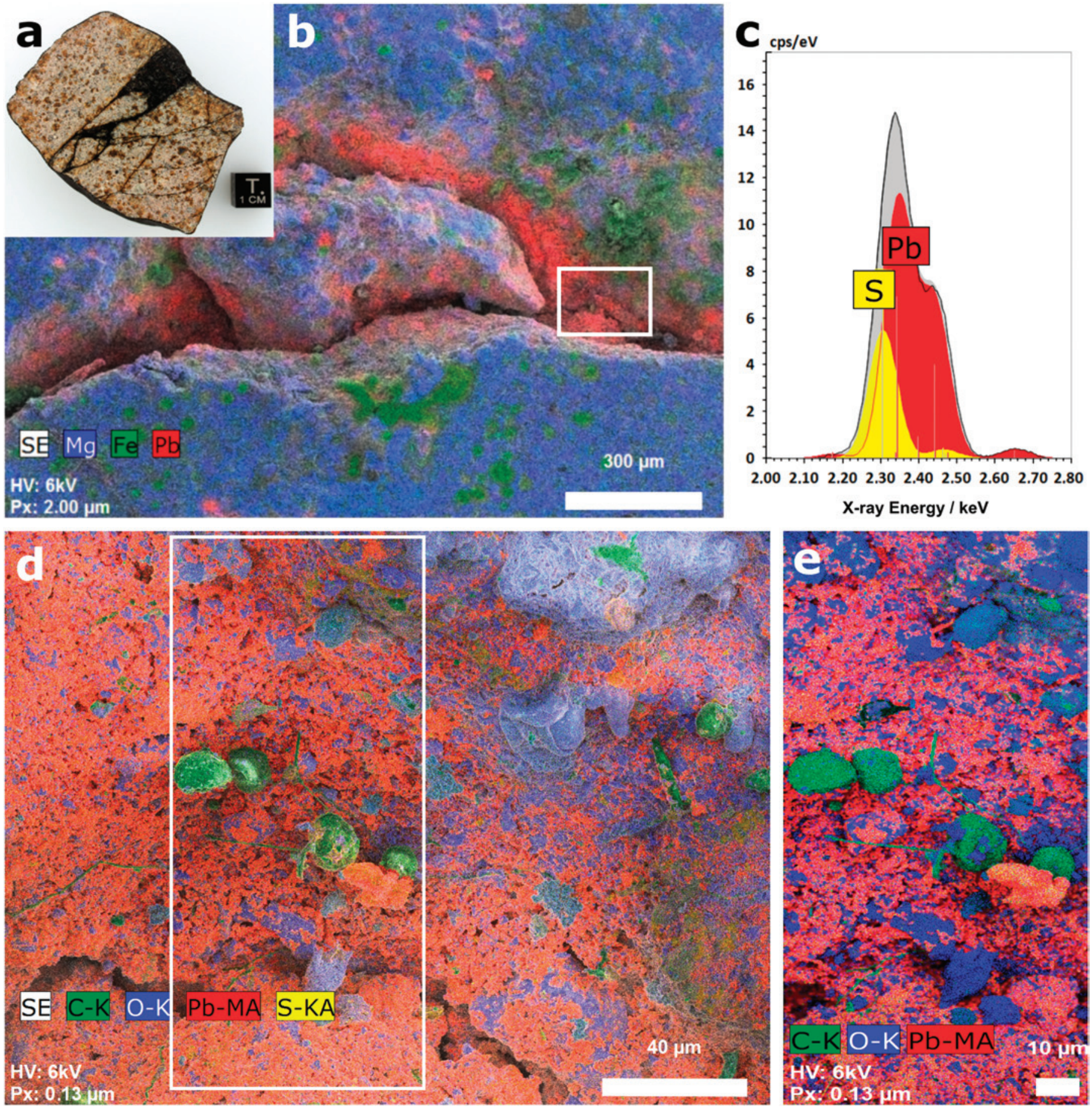

Figure 2: Historic Mocs meteorite. (a) Photograph of the sample $(65 \mathrm{~mm} \times 50 \mathrm{~mm})$. (b) Overview X-ray map at $6 \mathrm{kV}, 10 \mathrm{pA}$, input count rate $3 \mathrm{kcps}$, and $800 \times 600$ pixels was acquired for $2800 \mathrm{~s}$. X-ray map was overlaid with the secondary electron (SE) image revealing lead contamination from the historical polishing process. (c) $\mathrm{Pb}$ M-line $(\mathrm{M} \alpha 2.343 \mathrm{keV})$ and S K-line $(\mathrm{K} \alpha 2.307 \mathrm{keV})$ were deconvoluted during measurement using a physical background subtraction and a least square fit with stored line profiles. (d) A higher magnification composite map showing only the elements $\mathrm{C}, \mathrm{O}, \mathrm{Pb}$, and $\mathrm{S}$ of the rectangular region of interest in (b) overlaid with the SE micrograph. This shows that lead and soot were deposited on the sample surface that may consist of sulfides (orange areas $=$ red + yellow) and oxides (blue shows the $\mathrm{O}$ content). (e) Image detail of the outlined area in (d), without S, documenting that carbon features $<300 \mathrm{~nm}$ in size (green filaments) can be made visible under high-vacuum conditions using low kV and ultra-low probe current. Sample courtesy of Natural History Museum, Vienna, Dept. of Mineralogy and Petrography. Sample NHMW-H9898 was a donation from industrial magnate Sigmund Sachsel. Special thanks to Ludovic Ferriere for taking the sample to the Bruker Nano laboratories for analysis and the resulting discussion.

probe currents, and the acquisition of high-resolution element mappings of large sample areas or of single nanoparticles with sizes down to a few $\mathrm{nm}$. Non-destructive sample analysis with high spatial resolution can be very difficult to accomplish using conventional EDS detectors because sample surface charging, electron beam damage, and shadowing effects are common problems in scanning electron microscopy (SEM) that complicate X-ray acquisition. 

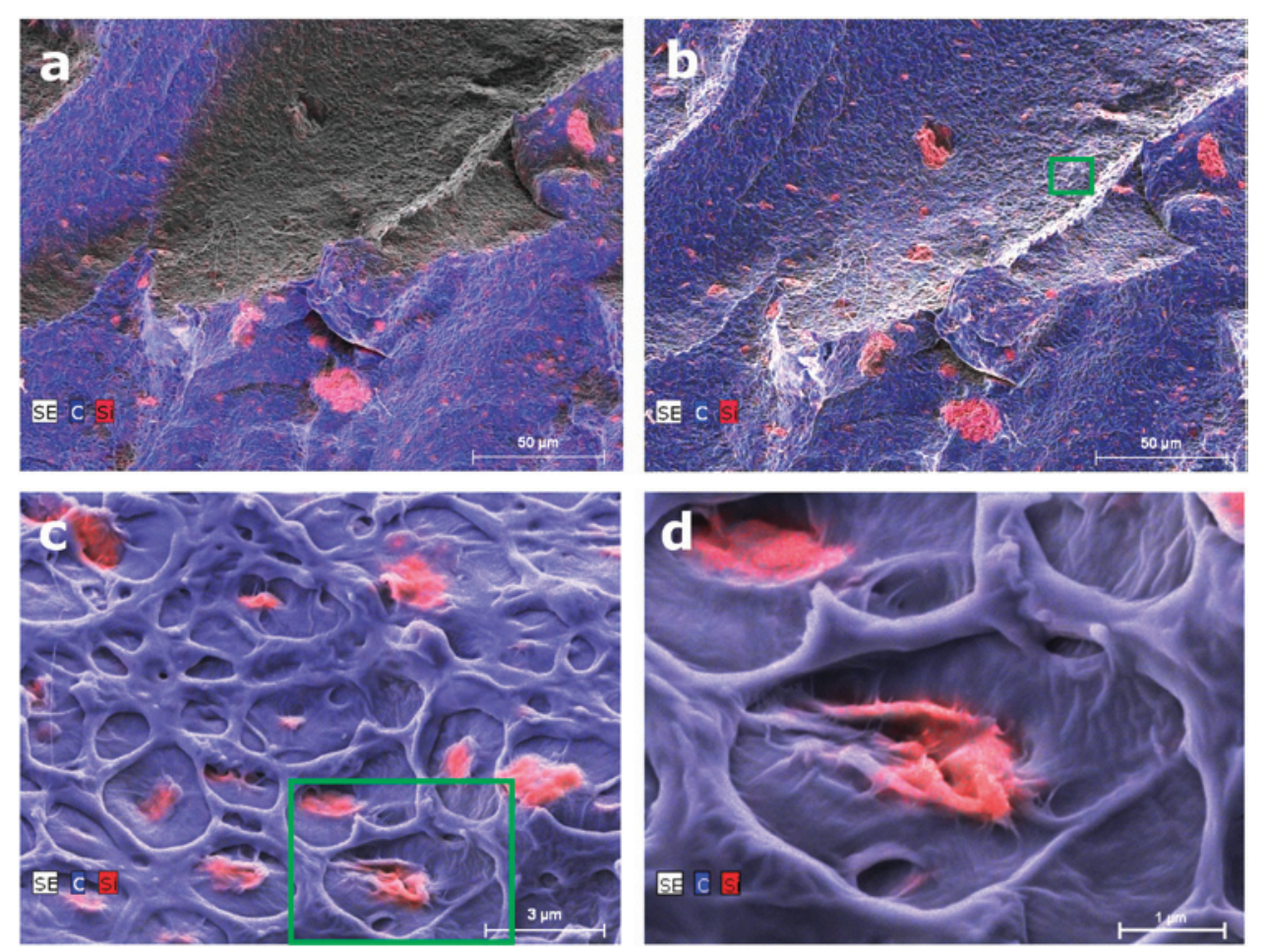

Figure 3: Combined C (blue) and Si (red) element maps overlaid with the corresponding SE images of a low-density polyethylene composite with silicon-containing organo-clay particles. The clay particles have formed large agglomerates. (a) Map acquired with a conventional $30 \mathrm{~mm}^{2}$ XFlash SDD setup, at $3 \mathrm{kV}, 220 \mathrm{pA}$, input count rate $0.8 \mathrm{kcps}$, $1024 \times 768$ pixels, acquired over $320 \mathrm{~s}$. Note the shadowing due to surface roughness. (b) Map acquired with the XFlash ${ }^{\circledR}$ FlatQUAD detector using the same acquisition conditions as in (a) but resulting in a 12.5 times higher count rate and no shadowing. (c) Magnified map area (green square) of map (b) in annular detection geometry. (d) Magnified map area (green square) of image (c) showing no shadowing effects. Sample courtesy of D. P. da Silva Dalto Center for Mineral Technology (CETEM), Brazil, M. J. O. C. Guimarães and M. E. F. Garcia, Federal University of Rio de Janeiro, Brazil [10].

Low accelerating voltages and low probe currents can be helpful for the analysis of electron-beam-sensitive materials and of nonconductive samples that charge during examination. Compared to low-vacuum analysis, often used to avoid sample charging, the use of low $\mathrm{kV}$ and low probe current in high vacuum circumvents the beam skirting effect. Beam skirting means that the spatial resolution of analysis is degraded by beam broadening because of collisions of probe electrons with gas molecules, atoms, and ions. Therefore low probe current under high vacuum conditions is desired. Thin TEM samples or life science samples producing a low $\mathrm{X}$-ray yield are additional situations well suited for analysis with this efficient annular detector. Its geometry was optimized in a way that allows the combination of thin-sample EDS with diffraction experiments in transmission, for example transmission Kikuchi diffraction (TKD) in the SEM [8]. Small particles and structures in the nanometer range also produce low X-ray yield, but they can be investigated at low accelerating voltages in SEM because of the small interaction volume attained. High-spatial-resolution EDS mapping on bulk specimens is best accomplished at low $\mathrm{kV}$ with the annular detector. Furthermore, fast mapping of sensitive samples and of large sample areas, including stitching maps together for complete analysis and statistics, are applications that benefit greatly from efficient X-ray collection. Last but not least, element mapping of porous or otherwise topographically structured samples present another application for the annular Bruker XFlash ${ }^{\circledR}$ FlatQUAD.

\section{Results}

High-vacuum EDS at low beam current of the historic Mocs Meteorite. This large meteorite fell as a bright ball of fire in February 1882 near the village of Mocs, Romania. A sample of this stony meteorite, officially named NHMW-H9898 (Figure 2a), entered the Natural History Museum Vienna (NHMV) collection in 1908 as a donation. A large surface of $60 \mathrm{~mm} \times 47 \mathrm{~mm}$ revealing a network of shock veins up to $10 \mathrm{~mm}$, cut and polished but uncoated, was investigated. For the current SEM-EDS investigation, a region of interest was identified after acquiring an 800 pixel by 600 pixel overview map at a $2 \mu \mathrm{m}$ by $2 \mu \mathrm{m}$ pixel size over 47 minutes (Figure $2 \mathrm{~b}$ ). The $\mathrm{Pb} \mathrm{M}$-line and S K-line were deconvoluted during measurement using a physical background subtraction and a least square fit to library spectra (Figure 2c). To avoid charging of the uncoated nonconductive meteorite surface, a $6 \mathrm{kV}$ beam with a probe current of less than $10 \mathrm{pA}$ was employed under highvacuum conditions to produce a second map with an input count rate of $3000 \mathrm{cps}$ (Figure 2d). The $6 \mathrm{kV}$ accelerating voltage kept the interaction volumes small for both electrons and X-rays. A smaller region of interest was chosen for an overnight map with a $130 \mathrm{~nm}$ pixel size, but otherwise had the same experimental conditions (Figure 2e).

The overview map of the Mocs meteorite (Figure 2b) shows lead enrichment in cracks that were most likely caused by an old polishing method employing a lead lapping plate that was used in the past. Sufficient data quality (large number of counts) allows deconvolution of the $\mathrm{Pb} \mathrm{M}$ and S K lines (Figure 2c), providing a separation of lead contamination from sulfide minerals that are part of the meteorite material beneath the contamination. The EDS analysis at higher spatial resolution (Figures $2 \mathrm{~d}$ and 2e) shows the deposition of lead on oxides and sulfides. Spherical carbon particles and filaments with sizes $<300 \mathrm{~nm}$ were observed, a sign of surface contamination with soot, very likely related to furnaces used at the NHMV during the last century or from the time before it was added to the NHMV collection.

Polymer with embedded organo-clay particles. Soft and porous materials are challenging not only in terms of beam sensitivity and charging but also in regard to judging the distribution of pores or property-enhancing particles. The following experiment describes the EDS analysis of a polymer compound containing Si-rich organo-clay particles, derived 
from organically modified montmorillonite. This new type of material was studied for its potential of improved mechanical, thermal, and flame-resistance properties $[9,10]$. For comparison of detector performance, element maps were acquired using the XFlash ${ }^{\circledR}$ FlatQUAD detector and a standard $30 \mathrm{~mm}^{2}$ XFlash ${ }^{\circledR}$ SDD under the same acquisition conditions at $3 \mathrm{kV}$ and $220 \mathrm{pA}$ beam current (Figure 3). The annular detector setup was optimized here not for highest solid angle but for highest possible take-off angle. So the working distance was increased above the optimum distance for large solid angle in order to investigate deeper pores. This still led to more than 12.5 times higher input count rate: $10 \mathrm{kcps}$ compared to the $0.8 \mathrm{kcps}$ for the conventional $30 \mathrm{~mm}^{2}$ SDD. Note that the result from the conventional SDD suffers from extreme shadowing effects because of the rough sample surface. Shadowing is nearly eliminated in the map acquired with the annular XFlash ${ }^{\circledR}$ FlatQUAD, allowing the microscopist to properly evaluate the distribution and embedding of the silicon-containing nano-clay particles in the polymer matrix.

Impact micro-crater in aluminum foil. Another fascinating analysis problem is the examination of artificial impact craters in aluminum foils produced in the laboratory under the collection conditions for NASA's

Stardust Mission. The primary task of this mission was to collect dust samples of a comet and return them to Earth for analysis. In order to find suitable instrumentation to analyze the precious samples from space, an analogous test experiment was created on earth [11]. Polymineralic aggregate projectiles were produced by aerosol impregnation of a dried slurry of olivine, diopside, and pyrrhotite powders. These projectiles were fired at $6.05 \mathrm{~km} \mathrm{~s}^{-1}$ from a light gas gun onto aluminum foils, forming impact micro-craters. The generated micro-craters were analyzed using a conventional SDD at $20 \mathrm{kV}$ and an annular XFlash ${ }^{\circledR}$ FlatQUAD at $6 \mathrm{kV}$ (Figure 4 ). Compositional analysis of the deeper part of the crater was not accessible using the conventional detector setup because of shadowing effects. In comparison, the analysis using the annular detector shows residues of the projectile that can be clearly distinguished from the aluminum target foil across the whole field of view without shadowing effects. Residues from the three mineral components were detected mostly in the crater interior. Silicate and sulfide melt with flow texture originating from olivine (Mg-green), pyrrhotite (S-blue), and diopside (Ca-red) were found. Mg-rich fragments, probably from olivine, occur as well as a few particles rich in Ca or S.

Titanium dioxide nanotubes decorated with gold nanorods. Titanium dioxide nanotubes attract attention in biomedicine because of their biocompatibility and bioactivity. It has been shown that they can promote the adhesion and proliferation of rat-bone-marrow-derived mesenchymal stem cells and hydroxyapatite nucleation and growth. Composite materials consisting of $\mathrm{TiO}_{2}$ nanotubes decorated with metal nanoparticles, for example, Au nanoparticles or Au nanorods, have an antibacterial effect that can be sustained in darkness. This effect is based upon the formation of a Schottky barrier between $\mathrm{TiO}_{2}$ and the conductive Au, which can set up a lethal reaction at the cell walls of the bacteria. Thus a porous $\mathrm{TiO}_{2}$ structure decorated with $\mathrm{Au}$ nanoparticles or nanorods is a promising composite material for orthopedic and dental implants [12]. Figure 5 shows the $\mathrm{Au}$ map of a $\mathrm{TiO}_{2}$ nanotube material containing Au nanorods of $6 \mathrm{~nm}$ to $12 \mathrm{~nm}$ in diameter. This Au X-ray map was acquired in 4 minutes at $5 \mathrm{kV}$ accelerating voltage and superimposed on the SEM image. The XFlash ${ }^{\circledR}$ 


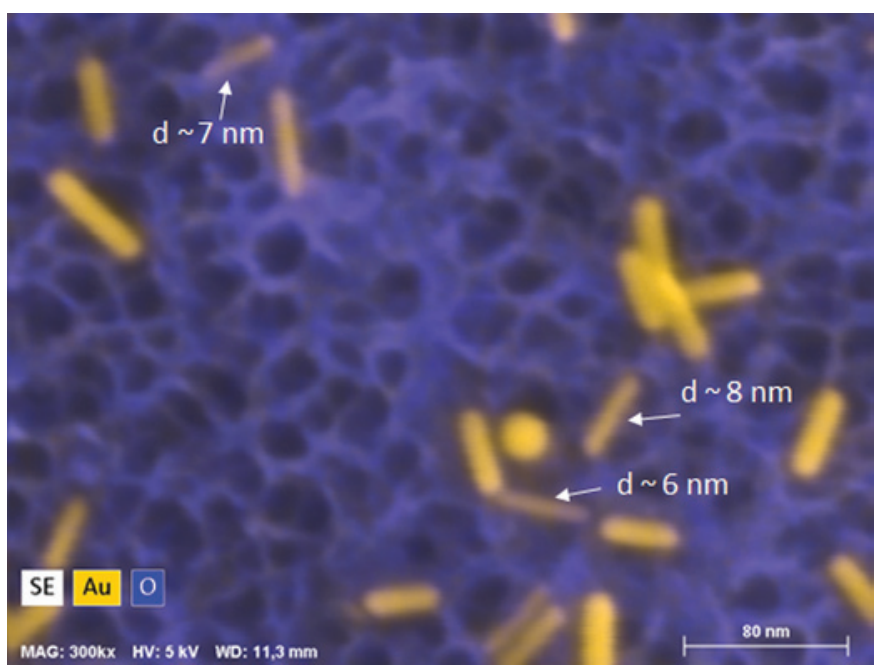

Figure 5: X-ray element map of the $\mathrm{O}$ and Au distribution in a Au-decorated $\mathrm{TiO}_{2}$ nanotube matrix, acquired using the XFlash ${ }^{\circledR}$ FlatQUAD, superimposed on an SEM image: $5 \mathrm{kV}$, input count rate $38 \mathrm{kcps}, 1024 \times 768$ pixels, acquisition time 240 s. Sample and data courtesy of Guangxi University, which also provided measurement time at the SEM, and special thanks to Prof. Xuanyong Liu and Prof. Yi Zeng from Shanghai Institute of Ceramics, Chinese Academy of Sciences, for generously providing the sample [12].

FlatQUAD with its high take-off angle and high X-ray collection angle allows measurement of the distribution, attachment, and size of the $\mathrm{Au}$ nanorods on the highly topographic $\mathrm{TiO}_{2}$ nanotube surface in one experiment. Furthermore, the $\mathrm{Au}$ nanorod distribution from the EDS element map could be correlated with data from fluorescence microscopy. This correlation shows which particular sites on this material attract bone mesenchymal stem cells and promote their spreading and which have antibacterial effects. This sample of Au nanorods on a porous $\mathrm{TiO}_{2}$-nanotube structure represents a type of TEM-suitable sample investigated here in the SEM; it shows the promise of highly sensitive EDS systems applied to STEM in SEM, so-called T-SEM EDS [13].

\section{Discussion}

The applications presented show that the annular XFlash ${ }^{\circledR}$ FlatQUAD detector can overcome some of the restrictions of conventional detectors. For the uncoated historic Mocs Meterorite, low $\mathrm{kV}$ and low beam current were necessary to avoid charging. This analysis is also possible with conventional detectors, but the large solid angle of the annular detector reduces the acquisition time significantly.

The same applies to samples where only small amounts of material are available for analysis, such as small particles or thin layers on bulk and electron transparent samples. Furthermore, light element samples produce low X-ray yields, so the annular design with four detectors is beneficial because it increases X-ray collection efficiency. Also, a low accelerating voltage may be used to produce a small interaction volume for high spatial resolution; again a high collection efficiency is important.

Energy-dispersive X-ray analysis in the cracks of the meteorite, in the porous polymer, and at the bottom of the impact microcrater is only possible because of the high take-off angle and signal acquisition from four different directions. There is a tradeoff between high take-off angle and solid angle. For a higher take-off angle the distance between sample and detector has to be increased. So using a take-off angle of more than $65^{\circ}$ reduces the solid angle from 1.1 sr to less than $0.5 \mathrm{sr}$ (Figure 1).

The signal acquisition, amplification, and processing of the four channels is completely separated. This increases the count-rate capability by a factor of four compared to the detector area of one segment. Three-dimensional information can be obtained using the different detector segments separately.

\section{Conclusion}

The annular XFlash ${ }^{\circledR}$ FlatQUAD detector is a device that combines a large solid angle ( $>1.1 \mathrm{sr}$ ) with output count rates up to 2.4 million counts per second. These properties can be used for efficient element mapping-applications such as ultra-fast mapping or large-area mapping. The collection efficiency allows elemental analysis of samples at both low accelerating voltages to achieve a small interaction volume and at low beam currents. These operating conditions provide high spatial resolution and high detection sensitivity without the necessity of applying a conductive coating or working at low vacuum. The high take-off angle annular detector arrangement enables element distribution mapping of highly topographic samples. Furthermore, use of this annular detector allows the analysis of small amounts of material. New analytical approaches in various fields become possible, especially in biological science and cultural heritage studies.

\section{References}

[1] L Strüder et al., Microsc Microanal 4 (1998) 622-31.

[2] JI Goldstein et al., Scanning Electron Microscopy and $X$-ray Microanalysis, Springer, New York, 2003.

[3] R Terborg and M Rohde, Microsc Microanal 17 (Suppl.2) (2011) 892-93.

[4] PG Kotula et al., Microsc Microanal 14 (Suppl.2) (2008) 116-17.

[5] NJ Zaluzec, Microsc Microanal 20 (2014) 1318-26.

[6] H Demers et al., Microsc Microanal 19 (Suppl.2) (2013) 364-65.

[7] H Soltau et al., Microsc Microanal 15 (Suppl.2) (2009) 204-05.

[8] RR Keller and RH Geiss, J Microscopy 245 (2012) 245-51.

[9] A Esfandiari et al., Journal of Applied Sciences 8 (2008) 545-61.

[10] DP da Silva Dalto, Master's thesis, Escola de Química, UFRJ, Rio de Janeiro, Brazil (2010).

[11] A Kearsley et al., 44th Lunar and Planetary Science Conference (2013).

[12] T Yang et al., Colloid Surface B 145 (2016) 597-606.

[13] D-V Hodoroaba et al., EMAS 2015; IOP Conf. Series: Materials Science and Engineering, 109 (2016) 012006-1; DOI: 10.1088/1757-899X/109/1/012006. 